\title{
Open source tools to support Integrated Coastal Management and Maritime Spatial Planning
}

\author{
Stefano Menegon ${ }^{1}$, Alessandro Sarretta ${ }^{1}$, Andrea Barbanti ${ }^{1}$, Elena Gissi ${ }^{2}$, and Chiara \\ Venier $^{1}$ \\ ${ }^{1}$ Consiglio Nazionale delle Ricerche, Istituto di Scienze Marine (CNR-ISMAR), Arsenale - Tesa 104, Castello \\ 2737/F, 30122 Venezia, Italy \\ ${ }^{2}$ Department of Design and Planning in Complex Environments, Università IUAV di Venezia, S. Croce 1957 - \\ 30135 Venezia Italy \\ Corresponding author: \\ Stefano Menegon ${ }^{1}$ \\ Email address: stefano.menegon@ismar.cnr.it
}

\begin{abstract}
This paper describes an open source suite of libraries and tools to support research activities on marine and coastal environment. The suite was initially implemented for the ADRIPLAN portal, an integrated web platform aimed at supporting Maritime Spatial Planning (MSP) activities and other activities concerning the managing of marine environment for the Adriatic-Ionian region. The main elements of the implemented solutions are: i) a GeoNode implementation for sharing geospatial datasets and maps; ii) a new python library (RectifiedGrid) that facilitates the work with geographical grid data; iii) a new python library (Tools4MSP) to perform spatial analysis and assessment of human uses, pressures and the potential impact of maritime and coastal activities on the environment; iv) a new GeoNode plugin (called GeoNode-Tools4MSP) that provides interactive widgets to set up the analyses and to visualize and explore the results.

The Tools4MSP and the developed software have been released as FOSS under the GPL3 license and are currently under further development.
\end{abstract}

Keywords: maritime spatial planning, open source, spatial analysis, geographic information systems, python, SDI, geoportals

\section{INTRODUCTION}

Management and planning of coastal and marine areas are complex processes that are more and more required to effectively support a coordinated development of socio-economic activities while preserving the environment using ecosystem-based approaches (European Union, 2014, Center for Ocean Solutions, 2011; Douvere, 2008). Practical tools to support the implementation of the various steps of Maritime Spatial Planning (MSP) have been developed in various contexts and also analysed to evaluate their usability for different purposes (Stelzenmüller et al., 2013).

A series of tools to support Ecosystem Based Management have been recollected and classified by the "EBM tools network" (https://ebmtoolsdatabase.org/), with respect to type, costs, skills, data and technological requirements. Considering as a central point of MSP the 
management of conflicts between marine uses, the project COEXIST (Stelzenmüller et al., 2013) developed a tool to analyse the level of coexistence among uses, depicting areas where different sectors more likely overlap in space and time.

Various authors proposed methodologies to create cumulative impact maps to reconnect the effects of human uses of the sea on environmental components, starting from the methodology firstly introduced by Halpern et al. (2008) at global scale, then implemented in several Marine Regions (Mediterranean by Micheli et al. (2013), Baltic Sea by (Korpinen et al., 2013), and North Sea (Andersen et al., 2013)). In particular, Stock (2016) developed an open source software for mapping human impacts on marine ecosystems.

The MSP process tends to involve several kind of users, from the data producers (e.g. domainexperts like ecologists and modellers) to the planners, who combine data and information to create a more comprehensive picture of a marine area and a reallocation of the human activities. Therefore, the availability of high quality geospatial data and informations from numerous fields (e.g. environmental sciences, tourism, human activities ad infrastructures) is a key issue of the MSP process. In this regard, the continuous development of Spatial Data Infrastructures (SDI) provides a favourable context for environmental management and planning Georis-Creuseveau et al. (2016)) while the importance of the integration of Geoportals in the context of SDIs has been highlighted by various authors (Maguire and Longley (2005)) and the role of a user-driven and community-based development is considered as fundamental for an effective and efficient use of the resource (De Longueville (2010) Georis-Creuseveau et al. (2016)).

This paper presents a set of web and open source tools developed to support the implementation of Maritime Spatial Planning in the Adriatic and Ionian Region, with a specific focus on the analysis of conflicts between marine uses and the analysis of cumulative impacts (CI) of human activities on marine environments. The whole system has been implemented with the ADRIPLAN Portal (http://data.adriplan.eu), the reference tool used to manage the data during the ADRIPLAN project (Barbanti et al., 2015)

The suite of tools composes a community-based integrated system where data are managed in a Spatial Data Infrastructure over the entire workflow, from the collaborative upload in a web portal, the creation of metadata, portrayal styles, aggregated maps, the set up of uses cases and the elaboration through specific modules producing final maps and descriptive reports.

\section{ARCHITECTURE AND IMPLEMENTATION}

In the view of integration of available software and development of new tools, the architecture described in Figure 1 has been developed. The schema highlights also the final users specifying their interaction with the system.

The core of the system is based on the GeoNode (http://geonode.org/) software (Fig. 11 - block 1): a django-based web platform for developing community-based spatial data infrastructures (SDI). GeoNode makes it easy to upload and manage geospatial data, to discover the resources and make content available via standard OGC protocols and to assemble web mapping applications.

The Tools4MSP (Fig. 1- block 2) are the main methodological tools developed and used in the ADRIPLAN project. The tools have been developed with the intent to carry out collaborative analyses and to improve the transparency of the whole analysis workflow. More in details, the 


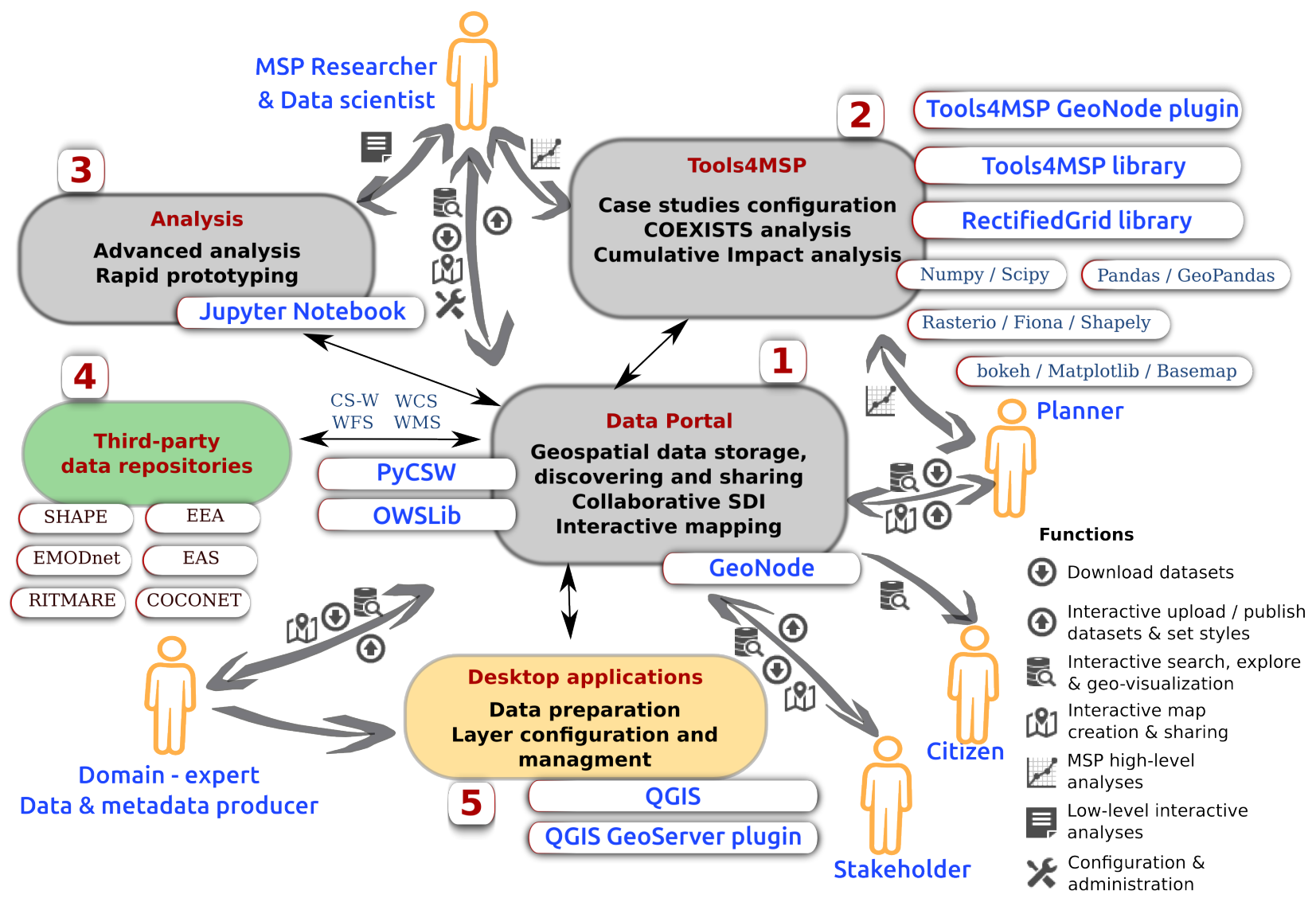

Figure 1. Simplified architecture of the ADRIPLAN portal for data management and analysis.

GeoNode-Tools4MSP plugin allows users to run experiments, simulations and analyses interacting directly with the data stored inside the Data Portal. The user can build several scenarios (called Case Studies) by choosing the analysis area, the data layers, the analysis grid cell and can produce and share the analysis results in terms of reports, tables, graphs and layers.

The core functionalities and analysis capabilities are implemented by the Tools4MSP library, a python stand-alone library able to operate independently from the GeoNode software. Currently, Tools4MSP implements the COEXIST analysis and the Cumulative Impact analysis but it's designed to be extensible and to allow the integration of other modules related with the MSP process like scenario planning and analysis, sectoral aspects (traffic, fishery, aquaculture, energy, etc.), socioeconomic aspects, participation processes.

RectifiedGrid is a lower level library which, combining into a single class several python packages (e.g. Numpy, SciPy, shapely, rasterio, fiona, geopandas, owslib, matplotlib-basemaps), simplifies geospatial grid-based analyses. Numpy is a notable package for scientific computing with Python with a support for large, multi-dimensional arrays and matrices: RectifiedGrid extends the numpy MaskedArray class by adding geospatial functionalities (i.e. projection awareness, bounding boxes). RectifiedGrid employs rasterio and fiona under the hood for raster and vector I/O and owslib to access data through OGC interoperable services. 

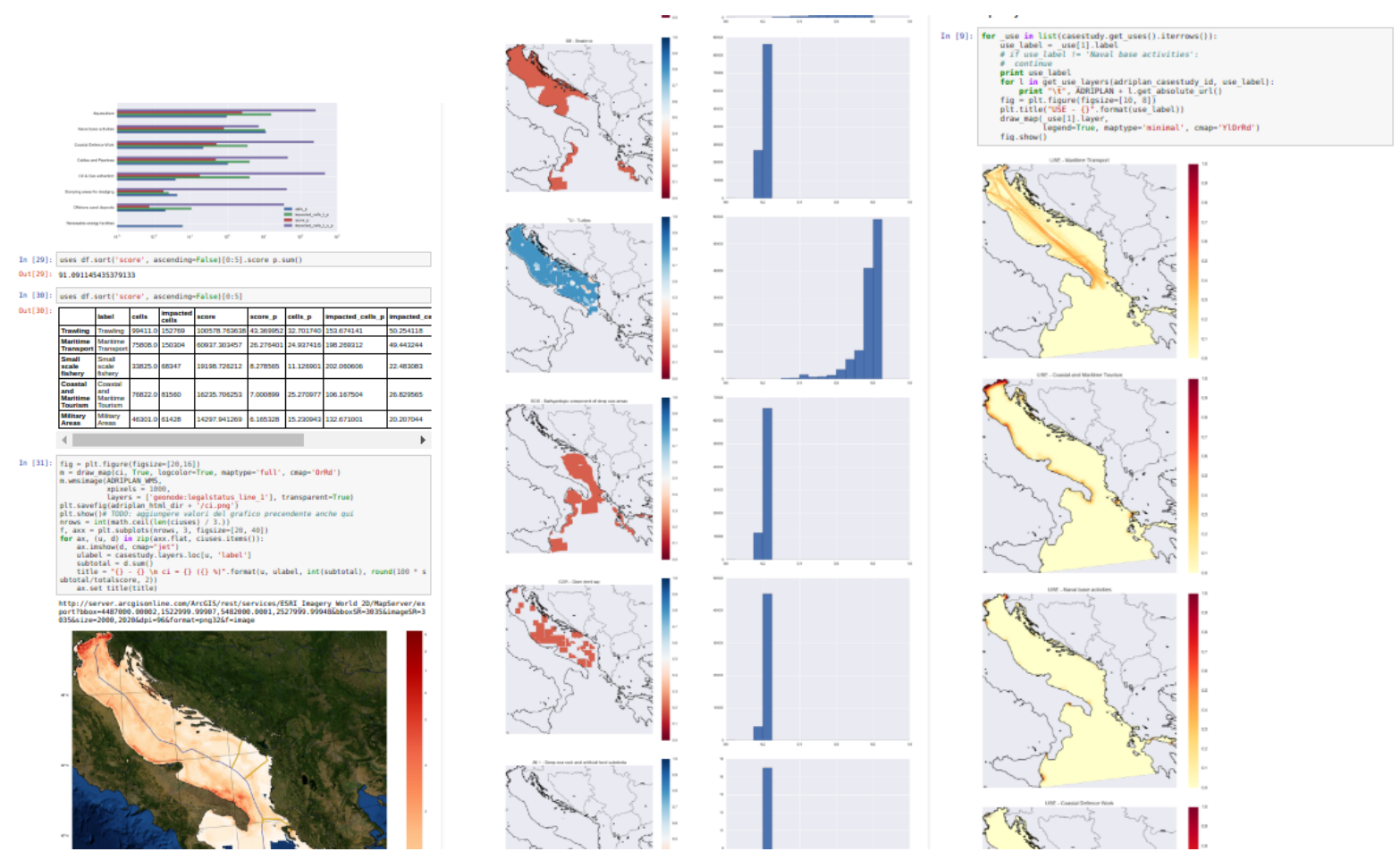

Figure 2. Example of low-level interactive analyses using Jupyter: case study for the whole Adriatic-Ionian region.

A low-level interactive analysis toolbox has been also implemented (Fig. 1- block 3) through Jupyter Notebook (https: / / jupyter.org/): a web-based interactive computational environment which allows a more flexible data exploration and analysis (see Fig. 2). Among other things, this toolbox fosters the rapid prototyping of new tools for MSP.

The block 4 of Fig. 11 (Third-party data repositories) highlights the ability of the system to integrate and make available data from other data portals and SDIs. The creation and maintenance of this network of collaborations allows the capitalization of existing multiple efforts and improves the availability of spatial datasets for users interested in MSP-related information. All these portals use interoperable OGC compliant web services to exchange spatial information.

The data publication and styling workflow are made simpler and more powerful through the use of specific GIS desktop applications (i.e. QGIS - QGIS Geoserver plugin - Fig. 1 block 5).

\section{Source code}

The software is available at the following repositories:

\section{RectifiedGrid}

Source code https://github.com/CNR-ISMAR/rectifiedgrid

\section{License GPL3}

\section{Tools4MSP}

Source code https://github.com/CNR-ISMAR/tools4msp 


\section{License GPL3}

GeoNode-Tools4MSP

Source code https://github.com/CNR-ISMAR/geonode-tools 4msp

\section{License GPL3}

\section{CONCLUSIONS}

We developed a set of open source tools to support the implementation of Coastal Zone Management and Maritime Spatial Planning in the Adriatic and Ionian Region. Three new python modules and libraries have been integrated to manage data over the entire workflow, from the collaborative upload in a web portal, to the creation of metadata, portrayal styles, aggregated maps, the set up of uses cases and the elaboration through specific modules producing final maps and descriptive reports. The integration of such a suite of open source tools allows a transparent, reproducible, highly interactive application of solid methodologies already applied with a specific focus on the analysis of conflicts between marine uses and cumulative impacts of human activities on marine environments. The integration of new modules will further improve the decision-support capabilities of the ADRIPLAN Portal. The products (maps, graphs, reports) are being used to support the development of maritime spatial plans within the implementation process of the MSP Directive (2014/89/CE) in various case study areas and marine waters in the Mediterranean sea. Furthermore, the release of the python tools under a GPL licence is a guarantee of possible future extensions and adaptation to other environmental domains and applications.

\section{ACKNOWLEDGMENTS}

This work was supported by: the Flagship Project RITMARE - Italian Research for the Sea, coordinated by the Italian National Research Council and funded by the Italian Ministry of Education, University and Research within the National Research Program 2011-2013; the ADRIPLAN (ADRiatic Ionian maritime spatial PLANning) project co-financed by European Commission DG MARE, call MARE/2012/25.

\section{REFERENCES}

Andersen, J., Stock, A., Heinänen, S., Mannerla, M., and Vinther, M. (2013). Human uses, pressures and impacts in the eastern north sea. Technical report, Aarhus University, DCE-Danish Centre for Environment and Energy.

Barbanti, A., Campostrini, P., Musco, F., Sarretta, A., and Gissi, E. (2015). Developing a Maritime Spatial Plan for the Adriatic Ionian Region. CNR-ISMAR, Venice, IT.

Center for Ocean Solutions (2011). Decision Guide for Selecting Decision Support Tools for Marine Spatial Planning. Technical report, The Woods Institute for the Environment, Stanford University. De Longueville, B. (2010). Community-based geoportals: The next generation? Concepts and methods for the geospatial Web 2.0. Computers, Environment and Urban Systems, 34(4):299-308.

Douvere, F. (2008). The importance of marine spatial planning in advancing ecosystem-based sea use management. Marine Policy, 32(5):762 - 771. 
European Union (2014). Directive 2014/89/EU of the the European Parliament and of the Council of 23 July 2014 establishing a framework for maritime spatial planning. O.J. L 257/135.

Georis-Creuseveau, J., Claramunt, C., and Gourmelon, F. (2016). A modelling framework for the study of spatial data infrastructures applied to coastal management and planning. International Journal of Geographical Information Science, pages 1-17.

Halpern, B. S., McLeod, K. L., Rosenberg, A. A., and Crowder, L. B. (2008). Managing for cumulative impacts in ecosystem-based management through ocean zoning. Ocean \& Coastal Management, 51(3):203-211.

Korpinen, S., Meidinger, M., and Laamanen, M. (2013). Cumulative impacts on seabed habitats: An indicator for assessments of good environmental status. Marine Pollution Bulletin, 74(1):311-319.

Maguire, D. J. and Longley, P. A. (2005). The emergence of geoportals and their role in spatial data infrastructures. Computers, Environment and Urban Systems, 29(1):3-14.

Micheli, F., Halpern, B. S., Walbridge, S., Ciriaco, S., Ferretti, F., Fraschetti, S., Lewison, R., Nykjaer, L., and Rosenberg, A. A. (2013). Cumulative Human Impacts on Mediterranean and Black Sea Marine Ecosystems: Assessing Current Pressures and Opportunities. PLoS ONE, 8(12):e79889.

Stelzenmüller, V., Schulze, T., Gimpel, A., Bartelings, H., Bello, E., Bergh, Ø., Bolman, B., Caetano, M., Davaasuren, N., Fabi, G., et al. (2013). Guidance on a better integration of aquaculture, fisheries, and other activities in the coastal zone: from tools to practical examples. Technical report, COEXIST project.

Stelzenmüller, V., Lee, J., South, A., Foden, J., and Rogers, S. I. (2013). Practical tools to support marine spatial planning: a review and some prototype tools. Marine Policy, 38:214-227.

Stock, A. (2016). Open Source Software for Mapping Human Impacts on Marine Ecosystems with an Additive Model. Journal of Open Research Software, 4(1). 